\title{
La guerra contra el lavado de dinero: ¿Puede ser exitosa sin menoscabar la confidencialidad y privacidad de aquellos que realizan transacciones legítimas?
}

José A. González Taboada

Universidad de Puerto Rico, Río Piedras

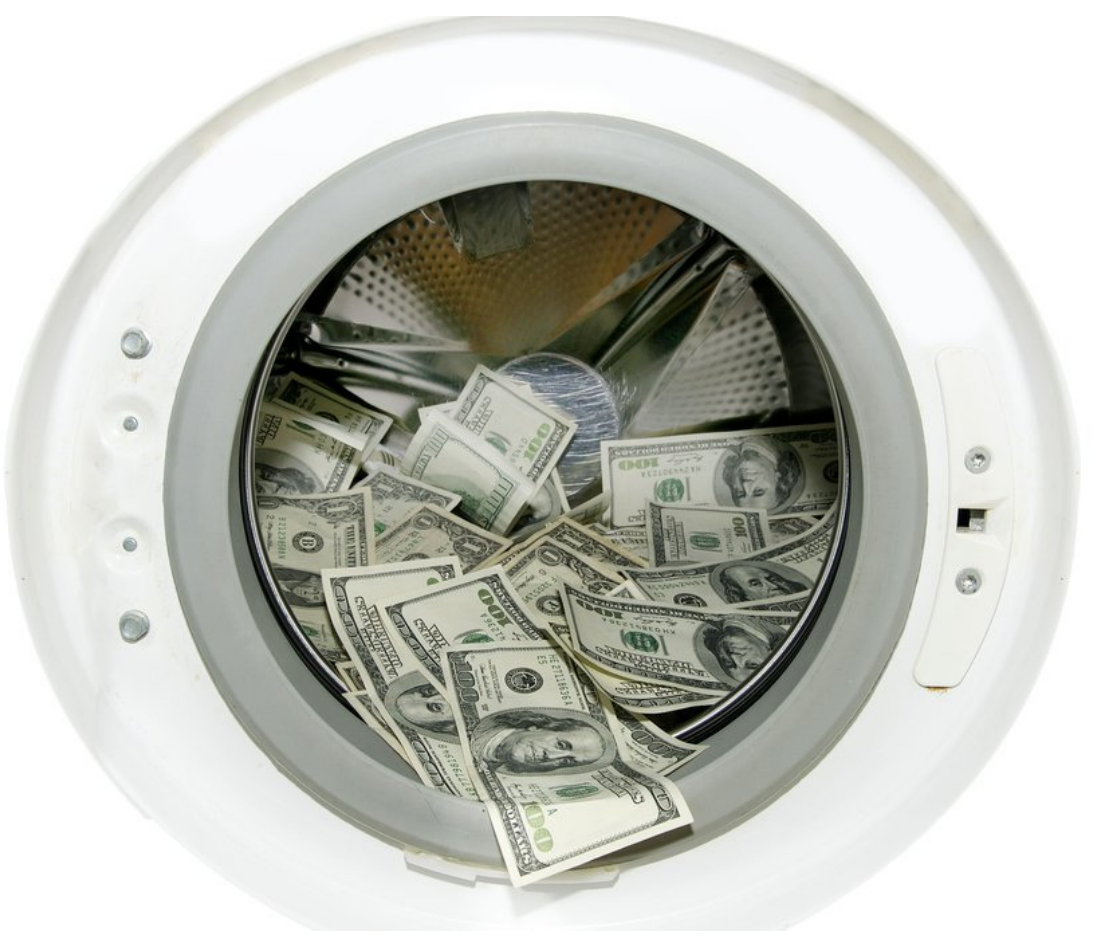

\section{RESUMEN}

El lavado de dinero no es una actividad delictiva aislada sino que está estrechamente relacionada con otras actividades delictivas como el terrorismo y el tráfico de drogas, entre otras. Estas actividades afectan a casi todos los países ya que corrompen los gobiernos y desestabilizan los sistemas financieros internacionales. El gran reto para las fuerzas del orden público es cómo prevenir el lavado de dinero sin menoscabar la privacidad y la confidencialidad que deben respetar las instituciones financieras. Es necesario educar a los profesionales del derecho, la auditoría y las finanzas en la materia del lavado del dinero para que puedan identificar aquellas transacciones que ameriten mayor escrutinio legal y financiero. Los gobiernos deben definir claramente las circunstancias en las que el derecho a la privacidad deberá ceder ante la necesidad de erradicar un mal social.

\section{PALABRAS CLAVES}

Lavado de dinero, delincuencia internacional, supervisión de sistemas bancarios, confidencialidad 


\section{Introducción}

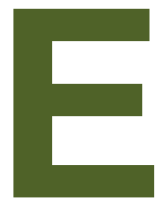

1 lavado de dinero no sólo es una actividad delictiva per se sino que, además, está estrechamente relacionado con otras actividades delictivas que preocupan a la comunidad internacional. El tráfico ilícito, bien sea de estupefacientes, armas de fuego, migrantes, mujeres, niños(as), obras de arte o armas nucleares, produce grandes cantidades de dinero a los grupos delictivos. Estos grupos tratan, entonces, de lavar el producto de sus actividades causando a su vez una cadena de delitos adicionales como la corrupción y la obstrucción a la justicia. Además, el lavado de dinero obstaculiza la detección de actividades criminales, provee nuevos recursos para expandir las actividades delictivas, distorsiona los mercados financieros y amenaza con destruir la actividad económica real de los países, sobre todo aquellos en proceso de desarrollo.

El lavado de dinero presenta un gran reto para los gobiernos porque éstos deben prevenir el que se utilice el sistema financiero para el lavado, sin obstaculizar la libre circulación del dinero y la prestación de servicios financieros. Otro reto es cómo supervisar los sistemas bancarios sin comprometer la confidencialidad y privacidad a la que tienen derecho aquellas personas naturales y jurídicas que realizan transacciones legítimas. Tanto las Naciones Unidas como la Unión Europea y los Estados Unidos han reconocido la gravedad de este problema y han tomado medidas para lidiar con el mismo. Este artículo resume la evolución y el estado actual del problema y presenta algunas recomendaciones respecto a la necesidad de educar a los profesionales de la contabilidad y otras disciplinas para que desarrollen conciencia del problema y las consecuencias de combatir el mismo.

\section{Primera Parte - La globalización, la delincuencia internacional y el lavado de dinero: tres temas entrelazados}

\section{La globalización y la importancia de los mercados financieros globales}

Dos eventos de gran impacto mundial aceleran la globalización en la última década del siglo XX. Uno de éstos fue la caída del comunismo y la disolución del bloque soviético en el este de Europa. Este evento provocó que muchos países comenzaran a transformar sus sistemas económicos, dirigiéndolos hacia el capitalismo y la libre empresa. El otro evento significativo fue la explosión de la tecnología en el campo de las comunicaciones, lo que facilitó la integración, paulatina unas veces, acelerada otras, de los sistemas financieros, creándose los mercados financieros globales.

Aunque las cifras varían ligeramente, tanto el Fondo Monetario Internacional, el Banco Mundial y la Agencia Central de Inteligencia estimaron el producto bruto doméstico del mundo entero en alrededor de $\$ 70$ trillones (como se define este término en Estados Unidos) en 2011 ${ }^{1}$. La cantidad de inversiones en los mercados de valores del mundo supera ampliamente dicha cifra. Los mercados de acciones solamente tenían un valor de $\$ 63$ trillones, de acuerdo con la Federación Mundial de Bolsas de Valores ${ }^{2}$. Algunos opinan que el poder de los mercados internacionales sobrepasa el de los líderes políticos y puede amenazar la democracia. Para compensar este lado negativo de la globalización financiera es necesario establecer un nuevo conjunto de

\footnotetext{
${ }^{1} \mathrm{http} / / /$ databank.worldbank.org/databank/download/GDP.pdf

2 www.world-exchanges.org
} 
reglas que asegure que las inversiones extranjeras ayuden, en lugar de perjudicar, a los países en desarrollo. Para lograrlo es imprescindible la cooperación de todos los países, especialmente aquellos que controlan el poder político y económico en el ámbito mundial. Pero estos esfuerzos se ven entorpecidos por otro fenómeno que se ha beneficiado de la globalización y de la explosión tecnológica en las comunicaciones: la delincuencia organizada internacional.

\section{La delincuencia organizada} internacional y sus efectos en la economía global

De acuerdo con un sicólogo jurídico colombiano (Díaz Colorado), el fenómeno de la delincuencia ha venido cambiando en la medida en que el mundo ha evolucionado y en la actualidad se observa una evolución de la criminalidad hacia una empresa altamente productiva y cada vez más sofisticada. Añade que los grupos de delincuencia se caracterizan hoy día por encontrarse en condiciones de actuar, tanto en la vertiente legal como en la ilegal de la actividad política y económica. Díaz Colorado estima que las tradicionales organizaciones criminales (llámense mafias, carteles o cualquier otro nombre) representan un volumen económico de un trillón de dólares y que la mitad de esta cifra se inyecta en el círculo financiero internacional con capacidad de producir rentabilidad. Schneider, en un estudio publicado en 2008 señala que en los 20 países más desarrollados el volumen de dinero lavado aumentó de $\$ 273$ billones en 1995 a $\$ 603$ billones en 2006. Puesto en otras palabras, la delincuencia organizada es hoy día una industria que no se fundamenta en delinquir por el delito mismo sino que busca la rentabilidad económica utilizando el delito como instrumento para conseguir sus metas.

Tan sofisticada es la delincuencia organizada internacional que Díaz Colorado la describe de la siguiente manera:

La delincuencia organizada actúa con criterios empresariales claramente establecidos, planificando sus actividades de acuerdo con los criterios económicos de la oferta y de la demanda, contemplando el impacto de la acción investigativa y penalizadora del Estado, situación que les permite regular el alza o la baja de precios. De igual manera, estructuran su actividad con la división del trabajo y la especialidad de la mano de obra. $^{3}$

¿Cómo afecta la delincuencia organizada a la democracia y a la economía global? La manera más eficaz es desestabilizando los gobiernos democráticos, particularmente aquellos con democracias incipientes, a través de la corrupción gubernamental. La corrupción obstaculiza seriamente el funcionamiento de los gobiernos y paraliza o desvía de sus objetivos a los procesos investigativos que tienen como función combatir la delincuencia organizada. Esto permite a los delincuentes organizados realizar sus fechorías con casi total impunidad. La estabilidad de los gobiernos se derrumba, además, porque pierde la confianza de la ciudadanía ya que ésta percibe que los funcionarios electos, las fuerzas armadas, la policía y otras agencias gubernamentales importantes están plagados de corrupción. Si

\footnotetext{
${ }^{3}$ http://www.psicologiacientifica.com/el-crimen-organizado/
} 
el pueblo no cree en las autoridades, no le brindará cooperación ni apoyo alguno.

El sistema económico también sufre graves daños porque el pueblo tampoco confía en las instituciones financieras privadas. Cada noticia de que algún banco importante ha sido señalado como participante, voluntario o involuntario, en un esquema de lavado de dinero mina la confianza del pueblo, no sólo en ese banco sino en la industria bancaria en general. En Puerto Rico, nuestro principal banco tuvo que pagar una multa de $\$ 21$ millones al gobierno de los Estados Unidos por fallar en alertar al gobierno sobre transacciones sospechosas que indicaban una alta probabilidad de lavado de dinero ${ }^{4}$.

\section{El lavado de dinero}

El lavado de dinero se define como el proceso mediante el cual alguien oculta la existencia o fuente ilegítima de ingreso y luego trata de hacerlo aparecer como legítimo. Por ejemplo, una organización puede realizar actividades delictivas en un país y adquirir un negocio legítimo en otro país. Se las arregla, entonces, para que los ingresos provenientes de sus actividades ilegales aparenten provenir del negocio legítimo. Muchas veces utilizan estrategias que no sólo pretenden lavar el dinero sino que además les permiten evadir las leyes de impuestos, violando las leyes desde diferentes ángulos. Dice Barbot:

Money laundering has clearly become endemic to our social, economic, and political frameworks; it ultimately affects and often subverts not only banking and other financial

\footnotetext{
${ }^{4}$ http://www.puertoricoherald.org/issues/2003/vol7n06/BancPopCanExpanden.html
}

institutions but also both small businesses and multinational corporations, legislators and law enforcement officers, lawyers and judges, politicians and high-ranking government officials, as well as newspaper and television media. In addition, money laundering erodes the income tax base of many nations, thereby creating fiscal policy problems. $^{5}$

Tal expresión de Barbot describe perfectamente el problema que representa el lavado de dinero y las consecuencias nefastas que tiene para los gobiernos democráticos, particularmente para las democracias incipientes. Por su parte, Rizkalla señala que el crecimiento del lavado de dinero se debe no solamente al aumento en la criminalidad sino también al aumento en la complejidad de las transacciones financieras y los avances tecnológicos. Con la rápida integración de los mercados mundiales y la remoción de barreras al libre flujo de capital, especialmente en la Comunidad Europea, se ha hecho más fácil ocultar el origen ilegal del dinero. Resulta irónico que los grandes adelantos tecnológicos, en los que muchos organismos policíacos han puesto su fe para combatir la delincuencia, sean simultáneamente el mejor aliado de los delincuentes. Es igualmente irónico que la aspiración de muchos países y muchas corporaciones legítimas, como la remoción de barreras al libre flujo del capital, se haya convertido en aliada de los grandes delincuentes internacionales.

El medio más usado por los que lavan dinero son las instituciones bancarias. Esto se debe a la multiplicidad de servicios que ofrecen los bancos y a las leyes que protegen la privacidad de los clientes de los bancos.

\footnotetext{
5 Tul. J. Int'l \& Comp. L. 161. P. 162.
} 
Pero cuando los bancos comienzan a tomar medidas para atacar el lavado de dinero, los ofensores buscan otros medios para lograr sus propósitos ${ }^{6}$. He señala varias otras formas que utilizan los que lavan dinero, entre éstas: instituciones de seguros, bienes raíces, loterías, "negocios" de exportación e importación, empresas fantasmas (front companies) y la Internet a través del dinero electrónico (e-cash). También señala He que algunos abogados y contadores se utilizan voluntaria o involuntariamente para lavar dinero $^{7}$. Mugarura afirma que los criminales utilizan a estos profesionales para darle visos de respetabilidad a las transacciones. Mientras más canales de lavado de dinero existan, más difícil se les hace a los gobiernos adoptar medidas para combatir esta actividad. Si añadimos que en muchos gobiernos hay funcionarios corruptos, las complicaciones son aún mayores ${ }^{8}$.

\section{Segunda Parte - La guerra contra el lavado de dinero: ¿la estamos ganando?}

Medidas en el ámbito nacional e internacional para combatir el lavado de dinero

La lucha contra el lavado de dinero se ha librado en muchos frentes nacionales e internacionales. De acuerdo con Rizkalla,

${ }^{6}$ Unger y den Hertog afirman: "Criminals seem to switch from the more controlled banking sector into still less controlled parts of financial markets, and from financial markets to other sectors." P. 287

7 Dice He: "Lawyers, accountants and other professionals provide a wide range of services such as creation of corporate vehicles, buying or selling of property or performing financial transactions. All of these operations are considerably vulnerable to money-laundering crimes.” P. 28

8 Dice Froomkin: "I am personally aware of three jurisdictions that laws in respect of banking, money laundering and off-shore business generally, were drafted by well-known money launderers." P. 5 los primeros esfuerzos europeos se remontan a la década de los 70 mientras que las Naciones Unidas comenzaron a atacar el problema en 1984. La convención de la ONU en aquel entonces estableció como meta proveer a los organismos policíacos internacionales las herramientas necesarias para minar el poder económico de los carteles. La convención de la ONU ha sido ampliamente aceptada en América Latina y en el Caribe. Está considerada como la base del régimen legal internacional en su lucha contra el lavado de dinero. Ha servido también de modelo para leyes más estrictas en todo el mundo.

En 1991 la Convención Europea sobre lavado de dinero adoptó una directiva con tres objetivos fundamentales: 1) prohibir el lavado de dinero en todos los estados miembros de la comunidad; (2) requerir de las instituciones financieras que faciliten las investigaciones criminales de las actividades de lavado, informando al gobierno las transacciones sospechosas y (3) reglamentar todas las profesiones que manejan grandes cantidades de dinero.

Las directrices a las instituciones financieras, tanto de la Convención Europea como de la ONU se pueden resumir en dos frases: "conozca su cliente" e "informe sobre toda transacción sospechosa". Increíblemente, el Banco Popular de Puerto Rico falló en informar las transacciones de un cliente a pesar de que este depositaba bolsas repletas de dinero en efectivo en una misma sucursal del banco, llegando a depositar más de \$20 millones. Algunos podrían argumentar que el término "transacción sospechosa" no está definido adecuadamente, pero en este caso de Puerto Rico es muy difícil aceptar dicho argumento. 
Una seria deficiencia con las medidas adoptadas en los años '80 y '90 del siglo pasado es que las mismas no están a la par con los recientes desarrollos tecnológicos. Uno de esos desarrollos es el dinero electrónico o cibernético (e-money). El dinero electrónico es un producto derivado de la Internet y de la demanda de los consumidores por eficiencia en el manejo de las transacciones financieras y de negocio. E-money facilita el lavado de dinero al utilizar entidades que no están reglamentadas por las leyes actuales, logrando que estas leyes sean ineficaces para combatir el lavado (Straub). Para prevenir el lavado cibernético, ante la realidad de una tecnología que evoluciona constantemente, los gobiernos deben reenfocar las leyes para que sea posible identificar las transacciones y se saque del anonimato a la "banca cibernética". El reto es realizar esto sin que las medidas que se implanten obstaculicen la innovación tecnológica que tanto beneficia a aquellos que hacen transacciones legítimas.

\section{La Financial Action Task Force $(\text { FATF })^{9}$}

FATF es una organización intergubernamental independiente fundada en 1989 por iniciativa de los países económicamente desarrollados (G7 en aquel entonces). Su misión es desarrollar políticas para combatir el lavado de dinero, el financiamiento del terrorismo y el financiamiento de la proliferación de armas de destrucción masiva.

Las primeras recomendaciones de la FATF para combatir el lavado de dinero datan de 1990. Las mismas se han revisado en varias ocasiones, siendo la más reciente en febrero de $2012^{10}$. Más de 180 países han

9 www.fatf-gafi.org

10 International Standards on Combating Money Laundering and the Financing of Terrorism \& endosado las cuarenta recomendaciones. Señalan la necesidad que los países implanten medidas que les permitan, entre otras cosas, evaluar riesgos, tomar medidas preventivas, incrementar la transparencia y facilitar la cooperación entre países. Estas recomendaciones se consideran estándares a seguir cuando se trata de combatir el lavado de dinero. Al mismo tiempo, la FATF mantiene un diálogo constante con el sector privado, la sociedad civil y otras partes interesadas en asegurar la integridad del sistema financiero mundial.

Un informe controversial de la FATF, de febrero de 2002, titulado Report on NonCooperative Countries and Territories, tenía el propósito de estimular acciones constructivas contra el lavado de dinero. El informe, como bien dice su título, señala aquellos países y territorios que la FATF considera que no están haciendo su parte en esta "guerra". La FATF amenazó con sanciones económicas a aquellos estados que se negaran a enmendar su "conducta". Para Doyle, este tipo de amenaza viola el derecho internacional al atentar contra la soberanía de muchos estados. Además, opina Doyle, la línea dura de la FATF viola la letra y el espíritu de la Carta de las Naciones Unidas y de los acuerdos internacionales que han surgido posteriormente. Imponer sanciones económicas puede ser altamente perjudicial para la guerra contra el lavado, y otros delitos internacionales porque se corre el riesgo que los países afectados se nieguen a continuar cooperando. Esto marcaría un retroceso en los esfuerzos internacionales de cooperación, los cuales han tomado décadas en lograrse.

Las Naciones Unidas y su guerra contra el lavado de dinero

Proliferation: The FATF Recommendations. Aparece en la web page de la FATF. 
La Oficina de Naciones Unidas Contra la Droga y el Delito (ONUDD) ${ }^{11}$, es un líder global en la lucha contra las drogas ilícitas y el delito internacional. Establecida en 1997, tiene el mandato de proporcionar asistencia a los estados miembros de las Naciones Unidas en su lucha contra las drogas ilícitas, el delito y el terrorismo. Entre otros proyectos, ha desarrollado, en colaboración con el Fondo Monetario Internacional, modelos de leyes, tanto para sistemas de derecho civilista como para sistemas basados en el derecho común.

En diciembre de 2005, ONUDD y el FMI publicaron su legislación modelo contra el lavado del dinero y el financiamiento del terrorismo, dirigida a países cuyo sistema de derecho es civilista ${ }^{12}$. En abril de 2009 publicaron la legislación modelo para países que aplican el derecho común ${ }^{13}$. Ambos modelos de legislación contienen un conjunto abarcador de medidas legales que debe contener la ley local para prevenir, detectar y sancionar eficazmente el lavado del dinero y el financiamiento del terrorismo, y que permita la cooperación internacional contra estos delitos.

\section{Parte 3 - Los problemas que causan las medidas contra el lavado a los sistemas financieros}

\section{Efecto en las instituciones financieras}

Doyle afirma que, históricamente, las sanciones económicas impactan negativamente a la población en general sin obtener los efectos deseados en la conducta

\footnotetext{
${ }^{11}$ En ingles, las siglas son UNODC.

12 http://www.unodc.org/documents/moneylaundering/2005\%20UNODC\%20and\%20IMF\%20M odel\%20Legislation.pdf

${ }^{13} \mathrm{http}$ ://www.unodc.org/documents/moneylaundering/Model_Provisions_2009_Final.pdf
}

de aquellos contra quienes están dirigidas las sanciones ${ }^{14}$. Otra preocupación seria con las medidas contra el lavado de dinero es el efecto que tienen éstas en las instituciones financieras. Duncan analiza este aspecto y concluye que, aunque por un lado las nuevas leyes sobre el lavado han creado conciencia en los bancos de la importancia de "conocer a sus clientes" y adoptar políticas que ayuden a prevenir y detectar el lavado de dinero, por otro lado estas leyes resultan en onerosas cargas económicas para los bancos, particularmente los bancos pequeños, según lo demuestra un estudio de Dolar y Shughart. La cantidad de nuevas reglamentaciones y la naturaleza de las mismas le imponen a los bancos pesadas cargas reglamentarias, precisamente en una época cuando los bancos, particularmente en los Estados Unidos, comenzaban a disfrutar de un período de desreglamentación luego de más de seis décadas de leyes altamente restrictivas.

La pregunta que se hace Duncan es si el costo de estas nuevas reglamentaciones y los cambios que han ocasionado en la forma de operar de los bancos son un precio razonable por los esfuerzos de mejorar la prevención y detección del lavado de dinero. Concluye Duncan que las nuevas leyes requieren de los bancos una carga investigativa para la cual no están equipados. Un banco, actuando como "juez y jurado", enfrenta metas en conflicto. Desea servir a sus clientes de manera rápida y eficaz, pero a la vez, no desea ser usado como vehículo para que los delincuentes internacionales laven su dinero. El banco realizará la transacción porque no

\footnotetext{
14 Algo similar dijo Alford en 1994: "Both domestic and international efforts to criminalize money laundering have placed increased regulatory burdens on commercial banks and other financial institutions, which are then passed on to customers through higher fees or lower interest rates." P. 439.
} 
quiere tomarse el riego de perder un cliente legítimo y simultáneamente informará la transacción a las autoridades. Esto, sin embargo, aumenta los costos del banco, los cuales pasa a sus clientes. Al final de cuentas, todos pagamos.

Los bancos necesitan adoptar sistemas mucho más sofisticados para enfrentarse a la delincuencia organizada internacional. Simultáneamente, las leyes anti lavado deben tomar en consideración la realidad de las operaciones bancarias y las necesidades de los clientes legítimos de los bancos. Si no se armonizan las metas conflictivas, será bien difícil alcanzar progreso en la guerra contra el lavado.

De acuerdo con Duncan, el que las leyes contra el lavado estén dirigidas principalmente a los bancos es un enfoque incorrecto porque la

Las leyes anti lavado (de dinero) deben tomar en consideración la realidad de las operaciones bancarias y las necesidades de los clientes legítimos de los bancos. Si no se armonizan las metas conflictivas, será bien difícil alcanzar progreso en la guerra contra el lavado. delincuencia

organizada está utilizando con cada vez más frecuentemente instituciones no financieras. Estas instituciones no se vigilan ni reglamentan de la misma manera que los bancos, lo que aprovechan los delincuentes para lavar allí su dinero. Los gobiernos deben redirigir sus esfuerzos hacia esas instituciones en proporción al volumen de lavado de dinero que ocurre mediante las mismas. En resumen, concluye Duncan, el reto que tienen los gobiernos y las agencias internacionales que combaten el lavado de dinero es doble: (1) comenzar a reglamentar instituciones a las que tradicionalmente no se les reglamenta porque los delincuentes internacionales las están utilizando para lavar dinero y (2) asegurarse que las políticas de reglamentación de los bancos comerciales y la carga que las mismas representan resulten en reducir la actividad de lavado de dinero y no la rentabilidad de los bancos.

La guerra contra el lavado de dinero y el derecho a la privacidad

Tomemos el problema de la privacidad del cliente. El objetivo del gobierno es prevenir el lavado de dinero, pero el objetivo del banco es proteger la confidencialidad de la información financiera de sus clientes. Esto es particularmente importante en aquellos países donde la constitución, explícitamente o por interpretación judicial, protege el derecho a la intimidad. Las leyes contra el lavado de dinero colocan al banco en la posición incómoda y costosa de decidir - caso por caso - si pesa más la expectativa de confidencialidad del cliente o la política gubernamental de prevenir el lavado. Si las leyes no proveen guías adecuadas y si no ofrecen al banco la protección e inmunidad necesarias, el banco no podrá tomar eficazmente esa decisión.

Uno de los artículos más elocuentes sobre el tema de la privacidad relacionado con las reglamentaciones dirigidas a prevenir el lavado de dinero fue publicado por Pasley en abril de 2002. El artículo señala que ha existido durante largo tiempo 
un ambiente tenso entre los que defienden el derecho a la privacidad, sobre todo la privacidad financiera, y la preocupación y los esfuerzos por prevenir el lavado de dinero. Aunque ha habido mucha legislación en esta área, la misma siempre ha sido complicada y controversial. Después de todo, se trata de un viejo dilema: cómo balancear la libertad individual y el orden social. Esto cobra mayor importancia y complejidad cuando el orden que se intenta preservar es uno de naturaleza internacional.

Es cierto, nos dice Pasley, que la privacidad es un derecho altamente valorado en los sistemas democráticos. Pero también es valorada la seguridad y la protección contra los males del lavado de dinero, actividad que financia, entre muchas cosas, el tráfico de drogas y el terrorismo. En estos tiempos de tensión e incertidumbre mundial, el lavado de dinero es uno de los problemas más críticos que enfrentan los gobiernos, pues no habrá manera de controlar la delincuencia internacional si no se logra cerrarle el acceso a las grandes sumas monetarias de dinero ilegal que circulan por los sistemas financieros de todo el mundo. Por tanto, parece ser necesario que los ciudadanos aceptemos un nivel mínimo de costos sociales y económicos para poder fortalecer a las agencias que luchan contra este mal.

Esto no será fácil de aceptar porque la información financiera de un individuo puede reflejar directamente su estilo de vida, sus intereses personales, sus afiliaciones políticas, la clase de literatura que lee, los grupos a los que pertenece, las causas que apoya y hasta los lugares que frecuenta. $Y$ es natural que el individuo desee que dicha información se mantenga privada.

Por otro lado, ya hemos discutido cómo el lavado de dinero amenaza con erosionar la integridad de los sistemas financieros, la estabilidad de las economías de los países y el valor de las monedas. Esto convierte al lavado de dinero, más que en un problema policiaco, en una seria amenaza para la seguridad nacional e internacional. Ante estos dos lados de la controversia, privacidad individual versus seguridad nacional e internacional, Pasley concluye diciendo que la búsqueda de un balance aceptable entre la preocupación por la privacidad y la necesidad de combatir este mal continuará evolucionando (p. 226).

\section{Conclusiones y recomendaciones}

El lavado de dinero es una de las peores amenazas que enfrentan los gobiernos en su lucha contra la delincuencia organizada internacional. Esto es así porque ninguno de los otros crímenes, tráfico de drogas, tráfico de personas, corrupción gubernamental y tráfico de armas y terrorismo podría realizarse si no producen dinero. Hoy los delincuentes organizados no cometen delitos ni retan a los organismos de ley y orden por diversión. O lo hacen con ánimo de lucro o lo hacen para financiar actos terroristas. Y para poder financiar las actividades $\mathrm{O}$ disfrutar de sus multimillonarias ganancias, necesitan hacer circular ese dinero a través de todo el mundo. Esto se consigue utilizando los mismos sistemas financieros que se crearon para servir a los ciudadanos honestos y a las corporaciones honestas.

En su esfuerzo por combatir el lavado de dinero, los gobiernos y las organizaciones internacionales han establecido leyes estrictas, las cuales afectan mayormente a los bancos, a pesar de que los delincuentes utilizan muchos medios para lavar el dinero. Estas leyes, además de aumentar los costos de operaciones y reducir las ganancias de los bancos, presentan un serio dilema. Los bancos tienen que colaborar con las 
autoridades, so pena de multas cuantiosas y otras sanciones legales y administrativas, sin obstaculizar las transacciones financieras legítimas y sin menoscabar la privacidad y confidencialidad de sus clientes. A medida que aumentan las tensiones como consecuencia del terrorismo y otros crímenes en el ámbito internacional, se hace más y más difícil establecer el balance correcto que requiere el dilema presentado. Puesto en otras palabras, las medidas contra el lavado del dinero son por un lado necesarias y por otro lado menoscaban el principio de libre empresa al obligar a las instituciones de negocios a recopilar y divulgar información sobre sus clientes; información que estas empresas no desean recopilar y mucho menos divulgar.

La solución a este problema no es sencilla y por eso, después de tantas décadas, no parece haber progreso. Sin duda, debe continuarse debatiendo este asunto en los altos foros nacionales e internacionales, atendiendo siempre ambos lados de la controversia. Pero igualmente importante es que se eduque a los profesionales $\mathrm{y}$ futuros profesionales del derecho, la contabilidad (particularmente la auditoría) y las finanzas en la materia del lavado de dinero para que estos estén capacitados para identificar aquellas transacciones que merezcan mayor escrutinio legal y financiero y las implicaciones de dicho escrutinio en la privacidad de los clientes. Las universidades, al educar a los estudiantes, los patronos en el adiestramiento continuo de sus empleados, y las asociaciones profesionales en el desarrollo de programas de educación continua para sus miembros, deben incluir cursos, seminarios y talleres sobre el tema del lavado del dinero y su impacto en la sociedad a través del mundo. Solo teniendo profesionales debidamente educados podremos progresar en la búsqueda de ese balance necesario entre combatir el crimen y proteger la privacidad de los individuos. La privacidad no es un derecho absoluto. Es necesario definir claramente las circunstancias bajo las cuales debe ceder en aras de un mayor bien social. De lo contrario, no ganaremos la guerra.

\section{Referencias}

Alford, D. (1994). Anti-Money Laundering Regulations: A Burden on Financial Institutions. North Carolina Journal of International Law \& Commercial Regulation, Summer, 19 N.C.J. Int'1 L. \& Com, Reg. 437.

Barbot, L. A. (1995). Comments: Money Laundering: An International Challenge. Tulane Journal of International and Comparative Law, Spring (3) Tul. J. Int'l \& Comp. L. 161.

Díaz Colorado, F. (2002) El Crimen Organizado. Recuperado de http://www.psicologiacientifica.com/el-crimenorganizado/

Dolar, B. \& Shughart, W. F. (2012). The USA Patriot Act's differential impact on large and small banks: Evidence from California's high-risk money laundering and related financial crime areas. Journal of Banking Regulation, 13(2). Apr. 127146.

Doyle, T. (2002). Cleaning Up Anti-Money Laundering Strategies: Current FATF Tactics Needlessly Violate International Law. Houston Journal of International Law, Winter (24) Hous. J. Int'l L 279.

Financial Action Task Force (FATF). (2012). International Standards on Combating Money Laundering and the Financing of Terrorism \& Proliferation - the FATF Recommendations.

Recuperado de http://www.fatfgafi.org/topics/fatfrecommendations/documents/fa tfrecommendations $2012 . \mathrm{html}$

Froomkin, S. M. (2003). Money Laundering, Corruption and the Proceeds of Crime - An International Reality Check.

Recuperado de http://www.docstoc.com/docs/32169177/MONEY -LAUNDERING_-CORRUPTION-AND-THEPROCEEDS-OF-CRIME---AN\# 
He, P. (2010). A Typological Study on Money Laundering. Journal of Money Laundering Control, 13 (1), 15-32.

Mugarura, N. (2011). The Institutional Framework Against Money Laundering and its Underlying Predicate Crimes. Journal of Financial Regulation and Compliance, 19 (2), 174-194.

Nixon, B. (2000). Global Forces: A Guide for Enlightened Leaders. Management Books.

Pasley, R. (2002). Privacy Rights V. Anti-Money Laundering Enforcement. North Carolina Banking Institute. April. 6 N.C. Banking Inst. 147

Rizkalla, A. (1998). Money Laundering: The European Approach. The Tulane European and Civil Law Forum, Winter (13) Tul. Eur. \& Civ. L.F. 111.

Schneider, F. (2008). Money Laundering and Financial Means of Organized Crime: Some Preliminary Empirical Findings. Venice University Center for Economic Studies. July. Recuperado de

http://papers.ssrn.com/sol3/papers.cfm?abstract id $=1136149$

Straub, J. (2002). Note: The Prevention of E-Money Laundering: Tracking The Elusive Audit Trail. Suffolk Transnational Law Review, Summer (25) Suffolk Transnat'1 L. Rev. 515.

Unger, B. \& den Hertog, J. (2012). Water always finds its Way: Identifying New Forms of Money Laundering. Crime, Law and Social Change, 57.3 Apr. 287-304.

United Nations Office on Drugs and Crime. (2011). Estimating Illicit Financial Flows Resulting from Drug Trafficking and other Transnational Organized Crimes - Research Report.

Recuperado de http://www.unodc.org/unodc/en/frontpage/2011/O ctober/illicit-money_-how-much-is-out-there.html

United Nations Office on Drugs and Crime. (2011). Model Legislation.

Recuperado

de http://www.unodc.org/unodc/en/moneylaundering/Model-Legislation.html?ref=menuside

\section{José A. González Taboada}

jose.gonzalez63@upr.edu

DBA, JD, CPA, catedrático y director del

Departamento de Contabilidad de la Facultad de Administración de Empresas del Recinto de Río Piedras en la Universidad de Puerto Rico. 